\title{
Spatial contrast sensitivity does not explain random figure discrimination latency
}

\author{
MICHAEL S. LOOP and TRUDY J. FREY \\ University of Alabama in Birmingham, Birmingham, Alabama
}

\begin{abstract}
Both humans and other mammals can more easily discriminate eight-sided figures from one another than they can figures having more or fewer sides. The hypothesis that this effect is based upon spatial contrast sensitivity was tested by varying viewing distance and hence spatial frequency content. Because viewing distance had little effect upon relative discrimination latency, it was concluded that the superior discriminability of eight-sided figures is not based upon spatial contrast sensitivity. The possibility that orientation tuning of visual cortex neurons may account for the effect is discussed.
\end{abstract}

Figures having eight sides are more easily discriminated from one another than are figures having more or fewer sides. Thus, human observers make a form discrimination more quickly (Brown, Hitchcock, \& Michels, 1962; Owen \& Brown, 1970) and against a higher background of visual noise (Crook, 1957) when figures have an intermediate number of sides. Because similar observations have been made for tree squirrels (Michels, Pittman, Hitchcock, \& Brown, 1962), squirrel monkeys (Nash \& Michels, 1966), and rhesus monkeys (Carlson, 1973), this phenomenon must be based upon some fundamental operating characteristic of the mammalian visual system rather than upon any cultural factors. In general, the effect has been accounted for by statements suggesting that few-sided figures are too simple to be unique and many-sided figures too unique to be simple (Brown et al., 1962; Carlson, 1973). Although this explanation is intuitively satisfying, it sheds little light upon the physiological basis of the phenomenon.

Considerable evidence exists that the mammalian visual system is most sensitive to intermediate spatial frequencies (Cornsweet, 1971). Psychophysically, this sensitivity is evidenced by lower contrast thresholds (Campbell \& Green, 1965), higher apparent contrast, within limits (Cannon, 1979), and faster reaction times to intermediate spatial frequencies (Harwerth \& Levi, 1978). Because the total highfrequency content of a scene increases as the number of contrast borders increases, the power spectrum of a scene will contain increasing high-frequency components as the number of edges (or figure sides) in-

This work was supported by National Eye Institute Grants R01 EY03303 and P30 EY03039. We thank Kristin Carlson for kindly sharing her stimulus figures and Caroline Dunn for manuscript preparation. The author's complete mailing address is: Department of Physiological Optics, School of Optometry/The Medical Center, University of Alabama in Birmingham, Birmingham, Alabama 35294. creases. Thus, superior discrimination of figures having an intermediate number of sides could be due to these figures' being composed of more intermediate spatial frequencies than are figures of more or fewer sides.

This potential explanation is highly attractive because it would account for a fairly mysterious result of form discrimination in terms of a first principle of visual psychophysics, namely, spatial contrast sensitivity. One obvious approach to this hypothesis would be to perform a Fourier analysis of the stimulus scenes (see Methods) or individual figures typically utilized. Such measurements are easily made by employing the optical Fourier transform (Goodman, 1968) technique and calibrating the resultant spectrum against a collection of sine-wave gratings of known contrast and spatial frequency. Although straightforward, this approach is inconclusive for two reasons. First, a preponderance of intermediate spatial frequencies in eight-sided figures would not necessarily indicate that spatial contrast sensitivity was responsible for their rapid discrimination. It would be necessary to vary spatial frequency, while holding figure structure constant, to establish spatial frequency's control over discriminability. Second, although much can be predicted from the spatial contrast sensitivity function about the detectability of complex waveforms at threshold contrast (Campbell \& Robson, 1968), rather less is certain at suprathreshold contrasts (Tolhurst, 1972). The situation is further complicated by the fact that multiple orientations are represented by the figures' sides. Thus, altough the Fourier spectrum of the stimulus scenes necessarily contains all the pieces of the puzzle, we know too little about how they fit to identify the relevant features of the picture.

Consequently, we have taken an alternative approach of varying the spatial frequency content of a collection of figures representing different "sided- 
ness" levels by varying the distance at which the figures are viewed. The rationale for this approach is that, if eight-sided figures are most rapidly discriminated over a wide range of viewing distances, then the absolute spatial frequencies represented by the stimulus figures, and hence the spatial contrast sensitivity, cannot account for the effect. This is true because doubling the viewing distance, for example, doubles the constituent spatial frequencies of all stimulus scenes.

\section{EXPERIMENT 1}

\section{Method}

Subjects. The 10 subjects ( 5 men, 5 women) were young adults of normal acuity who worked in the School of Optometry.

Stimuli. Stimulus figures were random figures derived according to the Method 1 rules of Attneave and Arnoult (1956). Twentyeight different figures were derived at six sidedness levels $(4,6,8$, 10,12 , and 14) and were equated for area using a photographic enlarger and planimeter. All stimulus scenes contained five identical (same) figures and one different (odd) figure selected from the same sidedness level. Within each sidedness level, figures were ordered by maximum extent, that is, longest axis, and stimulus scenes were constructed from the figures having the greatest and second-greatest extent, the figures having the second and thirdgreatest extent, and so forth. Thirty-six different stimulus scenes were used at each sidedness level, resulting in a total of 216 stimulus scenes.

A stimulus scene contained five same and one odd figure arranged in two rows. Each figure's longest axis was oriented vertically. High-contrast $(93 \%)$ positive (LPD-4) slides were made of each stimulus scene and projected (Buhl QBC projector) onto a viewing screen, which resulted in black figures on a white (280$\mathrm{cd} / \mathrm{m}^{2}$ ) background. At the closest viewing distance $(2.74 \mathrm{~m})$, one figure subtended about $2 \mathrm{deg}$ and the whole display was $11.6 \times$ 7.9 deg.

Apparatus and Procedure. The subject's response panel contained six red response push buttons arranged in two rows. Below the response buttons was a green stimulus presentation push button that displayed a stimulus scene for the duration of a press by activating a fast (Uniblitz $25 \mathrm{~mm}$ ) mechanical shutter. The duration of a stimulus scene presentation was measured by a photocell and electronic timer accurate to $1 \mathrm{msec}$.

The subject's task was to present each stimulus scene for as long as it took to locate the odd figure, release the shutter button, and press the red response button whose position on the panel corresponded to the position of the odd figure in the stimulus scene. The subject was instructed to be correct and quick. Each subject was given sufficient (20) practice trials to become comfortable with the procedure. Correct and incorrect responses were signaled to the subject by different tones, and the enforced intertrial interval was $3 \mathrm{sec}$. All trials were initiated by the subject.

The sequence of stimulus scenes was arranged so that every 12 slides each of the six possible positions of the odd figure was used twice and each sidedness level was used twice. Each subject was tested on two occasions. During each session a subject viewed half the slides at $2.74 \mathrm{~m}$ (near) and half the slides at $5.48 \mathrm{~m}$ (far). Which viewing distance was used first during a session was counterbalanced within and between subjects. Thus, each subject viewed each stimulus scene once at both viewing distances.

\section{Results}

Each subject's data were analyzed to determine mean discrimination latency at each sidedness level. Trials that resulted in errors $(3 \%)$ were excluded from the analysis. Trials that elicited latencies longer

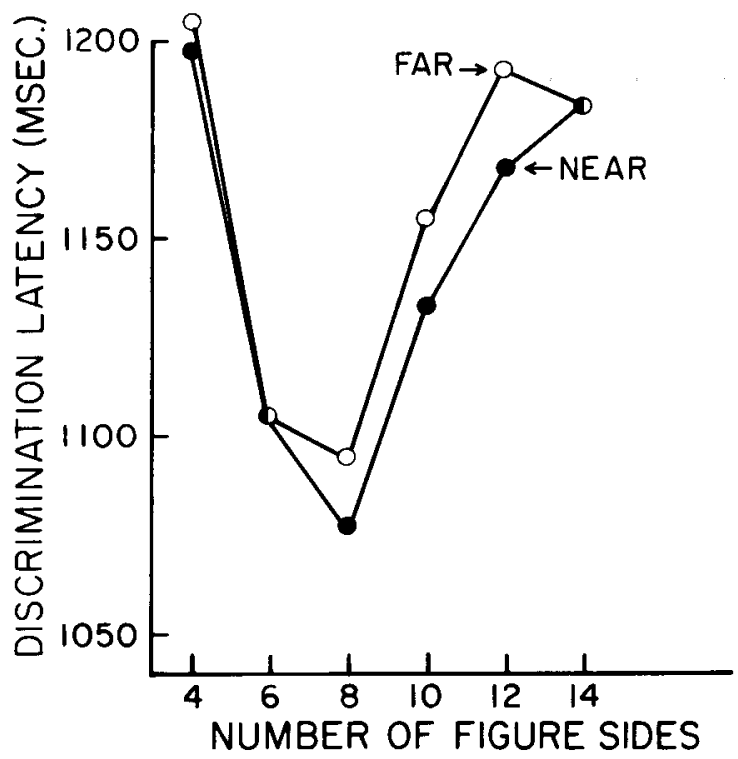

Figure 1. Mean discrimination time as a function of sidedness level for two viewing distances: Near (solid circles, $2.74 \mathrm{~m}$ ) and far (open circles, $5.48 \mathrm{~m}$ ).

than $3,500 \mathrm{msec}(.3 \%)$ were assigned a latency of $3,500 \mathrm{msec}$. Figure 1 illustrates mean discrimination latencies at both viewing distances as a function of sidedness level. An analysis of variance of mean discrimination latencies showed that eight-sided figures were discriminated most rapidly $[F(5,45)=12.28$, $p<.0001$ ]. However, neither the effect of viewing distance $[F(1,9)=.23]$ nor the interaction of sidedness level and viewing distance $[F(5,45)=.25]$ was statistically reliable.

The relationship between errors and sidedness level mirrored the discrimination latency function; although the overall error rate was low, $3 \%$, it ranged from $1.3 \%$ for eight-sided figures to $6 \%$ for foursided figures.

\section{EXPERIMENT 2}

The possibility was pointed out to us that the oneoctave variation in spatial frequencies effected by doubling the viewing distance might not be sufficient to reveal an influence of absolute spatial frequencies. Compatible with this possibility was the fact that there was no effect of viewing distance upon discrimination latency even though spatial frequency is known to influence detection latency (Harwerth \& Levi, 1978). This latter detail could be explained by other offsetting factors, such as smaller stimulus scenes' requiring fewer eye movements and/or falling mostly within the fovea. Nonetheless, we conducted a second experiment, on an entirely new population of subjects, in which viewing distance was varied over an eightfold range, thus spanning three octaves of 
spatial frequency. In this second experiment, the near viewing distance was equivalent to half the near viewing distance of Experiment 1, and the far viewing distance was equivalent to double the far viewing distance of Experiment 1.

\section{Method}

Subjects. The 12 subjects ( 7 men, 5 women) were young adults selected from the School's sophomore class. All had visual acuity correctable to $20 / 20$.

Apparatus and Procedure. The apparatus and procedure were identical to those of Experiment 1 in all but two respects.

First, so as to make the new viewing distances equivalent to half the near and double the far distances of Experiment 1 (and yet still conduct the experiment indoors), stimulus scenes were rear projected onto a screen (3M, Glass-Cat) and viewed from $.68 \mathrm{~m}$ (near) and $5.44 \mathrm{~m}$ (far). Thus, at the near distance, the stimulus scenes subtended a visual angle of $23.2 \times 15.8 \mathrm{deg}$, and at the far distance, $2.9 \times 2 \mathrm{deg}$.

Second, each subject viewed all stimulus scenes at both viewing distances in one 60-min session. Half the subjects were tested first at the near viewing distance and then at the far viewing distance, and half in the opposite order.

\section{Results}

Each subject's data were analyzed to determine mean discrimination latency at each sidedness level. Trials that resulted in errors $(2.2 \%)$ were excluded from the analysis, and latencies longer than $5,000 \mathrm{msec}$ $(.3 \%)$ were assigned a latency of $5,000 \mathrm{msec}$. This long-latency criterion was different from that in Experiment 1 because more subjects more often exceeded 3,500 msec (always at the far viewing distance). Figure 2 illustrates the mean discrimination latencies at

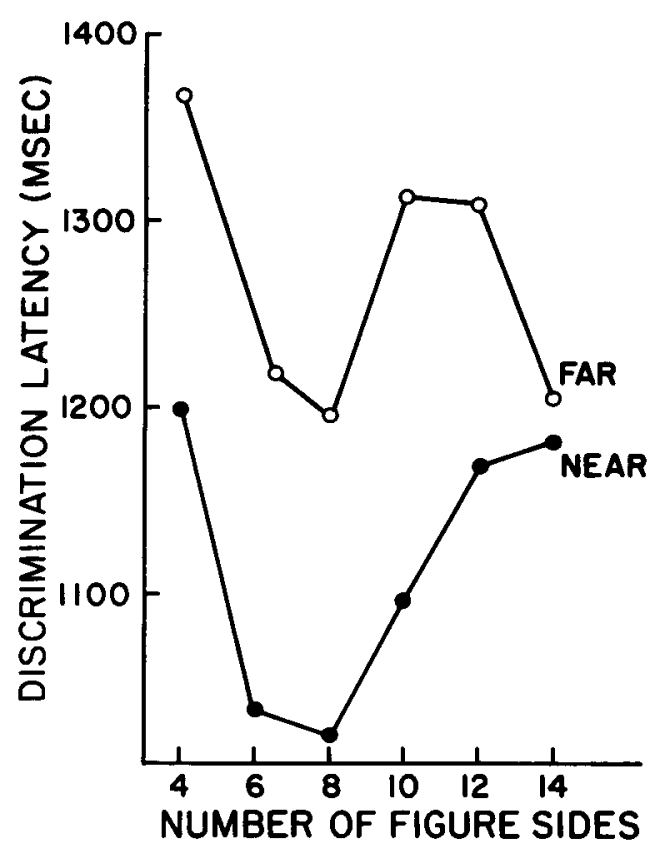

Figure 2. Mean discrimination time as a function of sidedness level for two viewing distances that are effectively half the near distance of Figure 1 and double the far distance of Figure 1. both viewing distances as a function of sidedness level. An analysis of variance indicated that the effects of sidedness level $[F(5,55)=12.24, p<.0001]$ and viewing distance $[F(1,11)=10.55, \mathrm{p}<.008]$ were statistically reliable, as was their interaction $[F(5,55)$ $=5.17, \mathrm{p}<.0006]$. As can be seen in Figure 2, eightsided figures were discriminated most rapidly at both viewing distances. The discrimination latencies of Experiment 2 (Figure 2) bracket those of Experiment 1 (Figure 1). That is, when the stimulus scenes subtended the largest visual angle (Experiment 2, near), discrimination latencies were the shortest, and when the stimulus scenes subtended the smallest visual angle (Experiment 2, far), discrimination latencies were the longest. Overall, except for the discrimination latency for 14-sided figures at the farthest viewing distance, the results of Experiment 2 are simply displaced in time (vertically) with respect to each other and to those of Experiment 1. The mean discrimination latency for 14-sided figures was much faster than for 10- or 12-sided figures and virtually identical to the mean discrimination latency for 8-sided figures at the farthest viewing distance. This aspect of the data accounts for the reliable interaction between sidedness and viewing distance. A reanalysis of the data, excluding the 14-sided figures at both viewing distances, indicated that both sidedness $[F(4,44)=13.97, p<.0001]$ and viewing distance $[F(1,11)=13.65, p<.003]$ exerted reliable influence but that their interaction did not $[F(4,44)=.79]$.

As in Experiment 1, the overall error rate was low, $2.2 \%$, and the same at both viewing distances. Eightsided figures were most accurately discriminated, $1.2 \%$ errors, and four-sided figures least accurately discriminated, $3.5 \%$ errors.

\section{DISCUSSION}

The discrimination latencies observed in this study are slightly faster than those observed by others (Brown et al., 1962; Owen \& Brown, 1970). This is probably due to the fact that our latencies are based primarily upon the time spent inspecting the stimulus, whereas in other studies discrimination latency included inspection time plus time taken to indicate the position of the odd stimulus.

As previous studies have reported (Brown et al., 1962; Owen \& Brown, 1970), discrimination latency is fastest for eight-sided figures. The present study confirms this phenomenon and demonstrates that it is immune to the influence of viewing distance and, hence, to absolute spatial frequency content of the stimulus scenes, over a three-octave range. From this we conclude that superior discrimination efficiency for eight-sided figures cannot be accounted for by their containing a preponderance of intermediate spatial frequencies to which human observers are most sensitive. 
At the same time, increasing the viewing distance did increase discrimination latency. Although viewing distance exerted a reliable influence only in Experiment 2, the results of Experiment 1 approximately split the difference between the near and far viewing distances of Experiment 2. The overall impression is that increasing viewing distance systematically increased discrimination latency without affecting accuracy. As noted earlier, several factors that covary with stimulus scene size might be expected to affect absolute discrimination latency. Which factors prevailed remains to be determined.

The decrease in discrimination latency for 14-sided figures when the stimulus scenes were the smallest (Experiment 2, far) is surprising. We believe this result to be a function of the stimuli, as opposed to an anomaly of the second group of subjects, for two reasons. First, the results of Experiment 2 (near) resemble the results of Experiment 1 (near and far) in the overall symmetry of discrimination latencies surrounding a minimum at 8 sides. Second, examination of the relative discrimination latencies for 10-, 12-, and 14-sided figures across all four curves suggests that, as stimulus scene size is reduced, the decrease in discrimination latency for 14-sided figures (Experiment 2 , far) is preceded by a decreasing difference in the discrimination latencies between 12- and 14-sided figures. Thus, the effect seems to appear systematically as stimulus scene size is reduced across both groups of subjects. It is interesting to note that, of the previous studies utilizing virtually identical procedures (Brown et al., 1962; Owen \& Brown, 1970), there is agreement as to the large influence of decreasing the number of figure sides below 8 but an apparent disagreement as to the magnitude of the effect of increasing the number of figure sides above 8. Owen and Brown (1970) observed discrimination latencies to be shortest for 8-sided figures and equally longer for 4 and 16-sided figures. Brown et al. (1962), on the other hand, found the increase in discrimination latency from 8- to 16-sided figures to be fairly small. Thus, the increasing discrimination latencies for figures of more than $\mathbf{8}$ sides does not appear to be a mirror image of the increased latencies elicited by figures of fewer than 8 sides.

Because the individual figures were not periodic, they did not contain any fundamental frequency. At the same time, all stimulus slides displayed six evenly spaced, equal-area patterns, such that the entire display was periodic but the same for all sidedness levels. Because the spatial frequency content of the stimulus scenes was not measured, we do not know exactly what was varied by changes in viewing distance. It seems clear, however, that absolute spatial frequency content cannot account for most rapid discrimination of eight-sided figures. We cannot speak to the question of whether or not relative spatial frequency content might hold the answer. In fact, it is certain that the phenomenon is based upon some fortuitous combination of spatial frequencies, orientations, and contrasts; there simply is nothing else in the stimuli.

The typical explanation, noted earlier, for the superior discriminability of eight-sided figures is unsatisfactory because it does not point to any physiological basis for the effect. In casting about for a testable alternative, we framed the question as follows: What makes two figures different if they have the same number of sides, identical area, and similar extent? The answer seems clear-orientation and length of sides. Cohen (1981) has shown that, if the odd figure in the display is simply a 180-deg rotation of the other five (same) figures, eight-sided figures are also most quickly discriminated. In this case, the only basis for discrimination is orientation of figure sides.

This line of thinking quickly leads to consideration of the neurons of the visual cortex, whose receptive fields are elongated and hence selective for the orientation of stimulus contrast borders (Hubel \& Wiesel, 1962, 1965). Although individual neurons are selective for particular orientations, every orientation is represented by some neurons. Therefore, if a stimulus that contains only a few orientations is viewed, it will presumably stimulate fewer cortical neurons than will a stimulus which contains more orientations. Thus, discrimination efficiency may increase as the number of figure sides increases, because more cortical neurons are stimulated as the number of orientations (sides) represented increases. However, because all cortical neurons respond to a range of orientations (Hammond \& Andrews, 1978; Wilson \& Sherman, 1976), a modest number of orientations may produce asymptotic recruitment of cortical neurons. The question then becomes why discrimination efficiency decreases with increasing number of sides past some optimum. Because the number of orientations represented by a figure increases as the number of sides increases, it is also true that the orientations represented in two different figures will become, on average, increasingly similar. Thus, as sidedness level increases, the discrimination between two different figures must be based upon decreasing differences in the orientations contained in the two figures and a decreasing number of cortical neurons, that is, those able to pick the differing orientations out of the noise of similar orientations. This line of argument leads to the conjecture that optimal sidedness level is determined by the orientation tuning of visual, presumably cortical, neurons. In so predicting, we have failed to consider some of the known physiological characteristics of cortical visual neurons, that is, that orientation tuning varies between cell types (simple or complex), cortical area $(17,18$, or 19), and representation in visual space (Hammond \& Andrews, 1978; Wilson \& Sherman, 1976). We see 
these details not as arguments against the hypothesis but rather as avenues of investigation to determine its validity.

\section{REFERENCES}

Atrneave, F., \& Arnoult, M. The quantitative study of shape and pattern perception. Psychological Bulletin, 1956, 53, 452-471.

Brown, D., Hitchcock, L., \& Michels, K. Quantitative studies in form perception: An evaluation of the role of selected stimulus parameters in the visual discrimination performance of human subjects. Perceptual and Motor Skills, 1962, 14, 5i9-529.

Campeell, F. W., \& Green, D. G. Optical and retinal factors affecting visual resolution. Journal of Physiology, 1965, 181, 576-593.

Campbell, F. W., \& Robson, J. G. Application of Fourier analysis to the visibility of gratings. Journal of Physiology, $1968,197,551-566$.

Cannon, M. W. Contrast sensation: A linear function of stimulus contrast. Vision Research, 1979, 19, 1045-1052.

Carlson, K. R. Visual discrimination learning of random figure problems by rhesus monkeys. Animal Learning \& Behavior, 1973, 1, 33-37.

Coнen, K. G. Influence of spatial frequency and figural complexity in visual discrimination performance. Master's thesis, 1981, University of Alabama in Birmingham.

Cornsweet, T. N. Visual perception. New York: Academic Press, 1971.

Crook, M. Facsimile-generated analogues for instrumental displays. In J. Wulfeck \& J. Taylor (Eds.), Form discrimination as related to military problems. Washington, D.C: National Academy of Sciences-National Research Council, 1957.
Goodman, J. W. Introduction to Fourier optics. New York: McGraw-Hill, 1981.

Hammond, P., \& Andrews, D. P. Orientation tuning of cells in areas 17 and 18 of the cat's visual cortex. Experimental Brain Research, 1978, 31, 341-351.

Harwerth, R. S., \& Levi, D. M. Reaction time as a measure of suprathreshold grating detection. Vision Research, 1978, 18, 1579-1586.

Hubel, D. H., \& WiEsel, T. N. Receptive fields, binocular interaction and functional architecture in the cat's visual cortex. Journal of Physiology, 1962, 160, 106-154.

Hubez, D. H., \& Wiesel, T. N. Receptive fields and functional architecture in two non-striate visual areas (18 and 19) of the cat. Journal of Neurophysiology, 1965, 28, 229-289.

Michels, K., Pittman, G., Hitchcock, L., \& Brown, D. Visual discrimination: Tree squirrels and quantified stimulus dimensions. Perceptual and Motor Skills, 1962, 15, 443-450.

Nash, A., \& Michels, K. Squirrel monkeys and discrimination learning: Figural interactions, redundancies, and random shapes. Journal of Experimental Psychology, 1966, 72, 132-137.

OWen, D., \& Brown, D. Visual and factual form discrimination: Psychophysical comparison within and between modalities. Perception \& Psychophysics, 1970, 7, 302-306.

TolHurst, D. J. Adaptation to square-wave gratings: Inhibition between spatial frequency channels in human visual system. Journal of Physiology, 1972, 226, 231-248.

Wilson, J. R., \& Sherman, S. M. Receptive-field characteristics of neurons in cat striate cortex: Changes with visual field eccentricity. Journal of Neurophysiology, 1976, 39, 512-533.

(Manuscript received October 19, 1981; revision accepted for publication March 19, 1982.) 